\title{
Philosophiques
}

\section{« La Minerve vient de faire sa physique »}

\section{Anne-Lise Rey}

Volume 44, numéro 2, automne 2017

Les nouveaux horizons du féminisme dans la philosophie francophone

URI : https://id.erudit.org/iderudit/1042332ar

DOI : https://doi.org/10.7202/1042332ar

Aller au sommaire du numéro

Éditeur(s)

Société de philosophie du Québec

ISSN

0316-2923 (imprimé)

1492-1391 (numérique)

Découvrir la revue

Citer cet article

Rey, A.-L. (2017). « La Minerve vient de faire sa physique ». Philosophiques, 44(2), 233-253. https://doi.org/10.7202/1042332ar

\section{Résumé de l'article}

L'article montre qu'après la " révolution scientifique » opérée par l'introduction des idées de Newton en France, Émilie du Châtelet a construit un dispositif épistémique inventif qui lui permet d'articuler principes métaphysiques et experimental philosophy. Je cherche à exposer que, s'il y a bien une relative invisibilité du travail philosophique d'Émilie du Châtelet dans l'historiographie des Lumières, cela tient à la fois au statut de femme savante à cette époque (Émilie Du Châtelet ne peut être réduite ni à une traductrice des textes de Newton, ni à une vulgarisatrice des thèses de Leibniz), mais aussi à la situation philosophique d'Émilie du Châtelet, qui ne peut se réduire à aucune filiation (cartésienne, leibnizienne ou newtonienne) et qui élabore une philosophie naturelle originale. 


\title{
«La Minerve vient de faire sa physique»'
}

\author{
ANNE-LISE REY \\ Université de Lille, \\ UMR STL/Vrije Universiteit Brussels²
}

\begin{abstract}
RÉSUMÉ. - L'article montre qu'après la «révolution scientifique » opérée par l'introduction des idées de Newton en France, Émilie du Châtelet a construit un dispositif épistémique inventif qui lui permet d'articuler principes métaphysiques et experimental philosophy. Je cherche à exposer que, s'il y a bien une relative invisibilité du travail philosophique d'Émilie du Châtelet dans I'historiographie des Lumières, cela tient à la fois au statut de femme savante à cette époque (Émilie Du Châtelet ne peut être réduite ni à une traductrice des textes de Newton, ni à une vulgarisatrice des thèses de Leibniz), mais aussi à la situation philosophique d'Émilie du Châtelet, qui ne peut se réduire à aucune filiation (cartésienne, leibnizienne ou newtonienne) et qui élabore une philosophie naturelle originale.
\end{abstract}

ABSTRACT. - This paper shows that after the "Scientific Revolution" stemming from the introduction of Newton's ideas in France, Émilie du Châtelet developed an innovative epistemic framework that allowed her to reconcile metaphysical principles with experimental philosophy. The author aims to show that the relative invisibility of Émilie du Châtelet's philosophical work within the historiography of the enlightenment is due both to her status as a learned woman at that time (Emilie Du Châtelet cannot be reduced merely to a translator of Newton's texts or a popularizer of Leibniz's theses) and to the philosophical position of Émilie du Châtelet, which cannot be reduced to a single philosophical affiliation (Cartesian, Leibnizian or Newtonian) and which allows her to develop an original account of natural philosophy.

Émilie du Châtelet a traduit les Principes mathématiques de la philosophie naturelle de Newton, et fait connaître la pensée de Leibniz en France dans ses Institutions de physique. Elle est ainsi bien souvent présentée dans l'histoire du $\mathrm{XVIII}^{\mathrm{e}}$ siècle comme une traductrice et une vulgarisatrice ${ }^{3}$. Il y a donc une forme d'opacité qui recouvre son travail proprement philosophique pour deux raisons principales qui sont à l'œuvre dans l'historiographie des Lumières.

1. Célèbre formule de Frédéric de Prusse à Jordan dans une lettre du 24 septembre 1740 : «La Minerve vient de faire sa Physique, il y a du bon, c'est Koenig qui lui a dicté son thème; elle l'a ajusté et orné par-ci par-là de quelque mot échappé à Voltaire à ses soupers. Le chapitre sur l'étendue est pitoyable, l'ordre de l'ouvrage ne vaut rien; il y a même de très grosses fautes, car dans un endroit elle fait tourner les astres d'Occident en Orient. Enfin, c'est une femme qui écrit et qui se mêle d'écrire au moment où elle commence ses études."

2. Ce travail s'inscrit dans le cadre d'un IF Marie Curie $n^{\circ} 748623$. Mon projet a pour titre EPISTOP pour «Epistemic Optimism».

3. S'y ajoute parfois la mention de son «amitié » pour Voltaire. 
Il faut tout d'abord mentionner ce que l'on pourrait appeler le stéréotype de genre ${ }^{4}$ : il existe toute une littérature qui se développe au XviII ${ }^{\mathrm{e}}$ siècle autour du motif de la marquise désireuse d'apprendre d'un philosophe toujours sensible à ses charmes. Il suffit de penser à l'Entretien sur la pluralité des mondes de Fontenelle ou au Newtonianisme pour les dames de Francesco Algarotti. Mais il faut sans doute également évoquer un ensemble de lettres ou de déclarations publiques dans lesquelles on s'étonne (et on se réjouit!) qu'une femme rende la philosophie naturelle si claire ${ }^{5}$. Il y a une donc forme d'ambiguïé sur la fonction même de vulgarisation.

Naturellement, Émilie du Châtelet jouera avec ce stéréotype à la fois dans sa correspondance lorsqu'elle s'interrogera (ou feindra de s'interroger) sur l'identité de la marquise au frontispice de l'édition du Newtonianisme pour les dames d'Algarotti, et quand elle mettra en scène dans l'avantpropos des Institutions de physique les conseils philosophiques qu'elle adresse à son jeune fils, il s'agira aussi d'inverser ce stéréotype de genre ${ }^{6}$. Il faut ajouter que d'autres éléments attestent qu'au cœur même du XvIII ${ }^{\mathrm{e}}$ siècle son travail philosophique était reconnu. En un sens, la dispute avec De Mairan paradoxalement en témoigne ${ }^{7}$, la traduction en allemand de son échange avec De Mairan sur les forces vives à l'initiative de Ch. Wolff également (ainsi que la traduction ultérieure des Institutions) ${ }^{8}$, son élection à

4. Florence Lotterie, Le Genre des Lumières, Paris, Classiques Garnier, 2013, p. Iо०I I 5 .

5. Lettre 566-4I 50 de Clairaut à Émilie du Châtelet, 4 janvier I74I in La correspondance d'Emilie du Châtelet (1740-1741), sous la direction de Ulla Kölving, Centre international d'études du XVIII e siècle, Ferney-Voltaire 20 I 8 (à paraître), p. 62: «Le premier livre m'a fait un grand plaisir en ce qu'il m'apprenait la métaphysique de Leibniz, au fait de laquelle je n'étais pas du tout. Je suis charmé de la connaître et je ne crois pas que personne me l'eût fait aussi bien entendre que vous. Je suis trop neuf dans cette matière pour vous en dire mon sentiment, mais ce que je puis bien assurer, c'est que si je pouvais être conquis à cette philosophie, ce serait par la façon dont vous la présentez. Quant à la partie physique de votre ouvrage, elle m'a fait beaucoup de plaisir aussi, non pas comme la première en m'ouvrant un champ nouveau, mais en me mettant sous les yeux dans un bel ordre et d'une façon agréable, les vérités les plus satisfaisantes de la physique.» Mais sous le compliment pointe la critique, puisque la lettre se poursuit en ces termes: "S'il m'était permis cependant de vous dire mon avis en entier, je prendrais la liberté de vous dire qu'il y a dans quelques endroits de petites négligences, dont quelques-unes peuvent venir de ce que vous avez voulu vous mettre plus à la portée de tout le monde, mais qui pourraient vous faire tort dans l'esprit des géomètres. "

6. Voir A.-L. Rey, «La littérarisation de la science newtonienne au XVIII ${ }^{\mathrm{e}}$ siècle: une littérature pour les dames?", Dossier «Littérature et Science: Archéologie d'un litige (xviexviii ${ }^{e}$ siècles) ", sous la dir. de Ph. Chométy et J. Lamy, Littératures classiques, n 85-20I4, p. 303-326.

7. Voir A.-L. Rey, "Agonistic and Epistemic Pluralisms: A New Interpretation of the Dispute between Emilie du Châtelet and Dortous de Mairan ", Paragraph, 40.I, Theories of Quarrels (A. Tadié, dir.), 20I7, p. 43-60.

8. Voir Lettre 332-46I0 d'Émilie du Châtelet à Christian Wolff du 22 septembre I74I, op. cit., p. I24-I25: "Je vous supplie aussi de vouloir bien y joindre un exemplaire de cette traduction allemande de ma dispute sur les forces vives, dont je vous ai l'obligation. Mon fils 
l'Académie des sciences de l'Institut de Bologne en I746 aussi, la reprise de son chapitre IV des Institutions de physique (loué par Euler dans une lettre de fin $\mathrm{I}_{74 \mathrm{I}^{9}}$ ) dans l'article «Hypothese (Métaphysique) ${ }^{10}$ » de l'Encyclopédie confirme enfin son importance et la diffusion de ses travaux.

On peut affirmer à partir de ces différents éléments qu'Émilie du Châtelet est, de son vivant, une femme savante reconnue, dont les travaux sont discutés par les grands savants de son temps avec lesquels elle entretient une correspondance régulière: que l'on songe simplement à Maupertuis, Euler, Clairaut ou encore Johann II Bernoulli. C'est donc sans doute l'historiographie des Lumières qui a conduit à occulter, ou parfois simplement à minorer, son importance dans l'histoire des sciences et de la philosophie des Lumières. En effet, peu de place est faite aux femmes savantes ou philosophes dans les histoires du XVIII ${ }^{\mathrm{e}}$ siècle.

Mais il y a sans doute une deuxième raison qui explique cette relative invisibilité des travaux d'Émilie du Châtelet dans l'histoire des sciences et de la philosophie du XVIII ${ }^{\mathrm{e}}$ siècle, c'est une lecture du XVIII ${ }^{\mathrm{e}}$ siècle français largement dominante qui suppose que, dans le champ de la philosophie naturelle, l'opposition centrale et structurante serait celle qui existe entre cartésiens et newtoniens. Cette option historiographique fait des cartésiens régnant à l'Académie royale des sciences de Paris d'archaïques métaphysiciens, aveugles à la science expérimentale, forcément moderne, qu'incarnerait Newton. De telle sorte que, par cette lecture en termes d'écoles de pensée irréductiblement opposées l'une à l'autre, on serait conduit à identifier les savants français du XVIII ${ }^{\mathrm{e}}$ siècle en fonction de leur appartenance à un «camp » bien déterminé: soit celui de Descartes, soit celui de Newton. Cette lecture en termes de filiations historiques est renforcée par l'idée que le $\mathrm{xvIII}^{\mathrm{e}}$ siècle marquerait le «triomphe ${ }^{11} »$ du paradigme newtonien.

apprend l'allemand, et je la lui ferai lire pour s'y fortifier. Dès que l'édition que l'on fait en Hollande des Institutions physiques sera finie, j'aurai l'honneur de vous en envoyer un exemplaire pour vous, et un pour celui qui veut bien avoir la bonté de les traduire.»

9. Lettre 602-4760 de Leonhard Euler à Émilie du Châtelet, fin I74I-début I742, op. cit., p. I45: «Mais surtout le chapitre sur les hypothèses m'a fait le plus grand plaisir, voyant que vous combattez, madame, si fortement et si solidement quelques philosophes anglais, qui ont voulu bannir tout à fait les hypothèses de la physique qui sont pourtant à mon avis le seul moyen de parvenir à une connaissance certaine des causes physiques. J'ai été souvent en peine, lorsque le discours roulait sur cette matière avec des Anglais, de trouver des raisons convaincantes pour bien faire voir l'utilité des hypothèses, mais je n'ai jamais pu développer mes idées sur ce sujet d'une manière si claire que vous. »

10. À la fin de l'article figure la mention suivante: "Voyez le chap. v. des Institutions de Phis. \& surtout le traité des Systèmes de M. l'Abbé de Condillac.»

11. Voir récemment encore, Mordechai Feingold, The Newtonian Moment, Isaac Newton and the Making of Modern Culture, The New York Public Library, Oxford University Press, 2004; Jonathan Israel, Les Lumières radicales, Paris, Éditions Amsterdam, 2005. 
Naturellement, cette lecture revient à ignorer la catégorie de «cartonian» forgée par Ellen Mc Niven-Hine ${ }^{12}$ pour décrire la position philosophique de Dortous de Mairan, à la fois cartésien et newtonien, et plus fondamentalement tout le contexte intellectuel français extrêmement complexe du premier XVIII ${ }^{\mathrm{e}}$ siècle que restitue avec minutie Carlo Borghero ${ }^{13}$ et qui montre, à l'instar de l'article "Newtonianisme " de l'Encyclopédie, la pluralité d'interprétations sous laquelle on comprend, à cette époque, le vocable de newtonien. À cela, il faut ajouter l'introduction de la philosophie de Leibniz en France qui vient troubler le jeu des oppositions rassurantes ${ }^{14}$.

La question pourrait donc se formuler en ces termes: comment rendre à nouveau visible non pas tant l'importance de la figure d'Émilie du Châtelet — ce travail est déjà en cours $^{15}$ —, mais sa singularité philosophique? Pour le dire autrement, comment démontrer que cette singularité est le signe d'une puissance philosophique?

Cela est possible en prenant quelques décisions méthodologiques et historiographiques. Autrement dit, il faut sans doute inclure dans le corpus qui permet d'apprécier la portée du travail d'Émilie du Châtelet les lettres qu'elle a échangées avec les savants et princes (Frédéric de Prusse) de son temps, les manuscrits non encore publiés (et récemment retrouvés) ${ }^{16}$ et les articles qu'elle a fait paraître dans les journaux savants (ou ceux qui mentionnent son travail) afin d'analyser dans le détail sa pratique philosophique.

Par ailleurs, d'un point de vue historiographique, il est extrêmement fécond de considérer qu'à côté des oppositions tranchées entre cartésiens et newtoniens s'est développé un courant de pensée, y compris après ce que l'on a appelé la «révolution scientifique », qui ne fait pas du newtonianisme

12. Ellen McNiven Hine, "Dortous de Mairan, the "Cartonian” ", Studies on Voltaire, 266, I989, p. I63-179.

13. Carlo Borghero, Les cartésiens face à Newton. Philosophie, science et religion dans la première moitié du XVIII siècle, Brepols, $20 \mathrm{II}$. Borghero montre, par exemple, comment le newtonianisme a pu être disqualifié en raison de sa proximité supposée avec le matérialisme (Cuenz), ou bien, selon une autre stratégie interprétative, considéré comme la suite de la « révolution » cartésienne (Gerdil).

14. Voir J. B. Shank, The Newton Wars and the Beginning of the French Enlightenment, Chicago, The University of Chicago Press, chap. VII «Leibnizianism and the French Enlightenment", p. 425: "This label [Leibnizianism] emerged after I 740 as an alternative to both Newtonianism and Cartesianism, yet its coherence (or lack thereof) was a product of the same contested and prismatic reception that had produced the other two philosophical "-isms" during the previous decades. The emergence of this third position also worked to complicate and to clarify the Newtonian/Cartesian binary in influential ways".

15. Voir le programme de réécriture d'histoire de la philosophie visant à intégrer des femmes philosophes dans une nouvelle histoire de la philosophie: http://www.newnarratives inphilosophy.net/ et, dans une perspective différente, les activités menées dans le «Center for the History of Women Philosophers and Scientists " à Paderborn.

16. L'histoire est connue. Un certain nombre de manuscrits d'Émilie du Châtelet ont été retrouvés en 20I2, mais dispersés à l'occasion d'une vente privée. Certains textes sont néanmoins à nouveau accessibles. 
une solution différente au problème du fondement métaphysique de la physique mais qui conçoit la possibilité d'articuler métaphysique et experimental philosophy. C'est en ce sens que je comprends cette formule d'Émilie du Châtelet à propos des Institutions dans lesquelles elle présente, entre autres, la physique de Newton: "[M]ais comme je suis persuadée que la physique ne peut se passer de la métaphysique sur laquelle elle est fondée, j'ai voulu lui donner une idée de la métaphysique de M. de Leibniz, que j'avoue être la seule qui m'ait satisfaite, quoiqu'il m'en reste encore bien des doutes ${ }^{17}$.» Pour Émilie du Châtelet, prendre acte de la révolution épistémique que constitue le newtonianisme ne signifie pas renoncer à la métaphysique et, en particulier, à celle qui lui semble la plus efficace: celle qu'elle désigne comme la métaphysique de Leibniz ${ }^{18}$.

Pour rendre raison de ce point relativement aveugle de l'historiographique des Lumières, j'ai forgé une catégorie heuristique qui ne correspond pas à une école de pensée: il s'agit du "leibnizo-newtonianisme ${ }^{19}$ ». Je n'entends pas le leibnizo-newtonianisme comme un courant de pensée, mais comme la mise en œuvre d'une combinaison effectivement présente, quoique souvent de manière différente, dans plusieurs textes importants des Lumières ${ }^{20}$. Il me semble donc que, pour peu qu'on prenne cette catégorie pour ce qu'elle est vraiment: un instrument de lecture des textes du $\mathrm{XVIII}^{\mathrm{e}}$ siècle et non le moyen de mettre au jour une tradition négligée, elle permet de restituer la complexité de la philosophie des Lumières qui n'est pas unilatéralement éprise du modèle empiriste newtonien.

En partant de ces réflexions, je voudrais montrer comment Émilie du Châtelet construit une philosophie naturelle originale en interrogeant et en discutant les conséquences métaphysiques de l'adhésion au principe de conservation, en particulier sur la question de la liberté, en I738, puis com-

17. Lettre 247, Émilie à Frédéric, avril I 740 (La correspondance d'Émilie du Châtelet, Ferney-Voltaire, éd. U. Kollving, à paraître, 20I 8 ).

18. Voir Sarah Hutton, «Between Newton and Leibniz: Emilie du Châtelet and Samuel Clarke, in Emilie du Châtelet between Leibniz and Newton, ed. Ruth Hagengruber, Springer, 20 I I. Voir p. 78 : «Nevertheless, her subscription to both Newton and Leibniz remains problematic from a modern perspective, especially in view of the way Newton and Leibniz are nowadays treated as leading representatives of the empiricist-rationalist schools, and therefore of rival British and German philosophical traditions. [...] Since Emilie du Châtelet's Institutions de Physique, assumes compatibility between Newtonian theories and Leibnizian metaphysics, it is reasonable to expect that her view of this controversy [the Leibniz-Clarke dispute] should hold clues about her understanding of the relationship of Leibniz's philosophy to Newton's. »

19. Voir «Le leibnizo-newtonianisme: la construction d'une philosophie naturelle complexe dans la première moitié du XviII ${ }^{\mathrm{e}}$ siècle. La méthode d'Émilie du Châtelet: entre hypothèses et experiences ", revue Dix-huitième siècle, dossier "La Nature ", juin 20I3, p. I I 5-I 29.

20. Que l'on pense à la démonstration expérimentale du principe de conservation des forces vives élaborée par Willelm's Gravesande dans son Introduction à la philosophie newtonienne ou encore à la volonté de dépasser Leibniz et Newton "en newtonien" dans la Theoria philosophiae naturalis de Roger Boscovich, pour ne donner que quelques exemples. 
ment les Institutions ont résolu ce problème, non pas grâce à la distinction wolffienne entre différents régimes d'intelligibilité de la substance comme le pensait Émilie du Châtelet en I 738, mais en choisissant une définition de la substance comme active. Cela lui a permis, par le dialogue qu'elle a mis en œuvre entre Leibniz et Wolff, de reconfigurer l'espace de la phénoménalité. Il me semble ainsi que s'esquissent les contours d'une philosophie naturelle exigeante.

\section{La liberté des forces vives}

Je voudrais partir d'un passage maintes fois cité d'une lettre à Maupertuis du 30 avril I738, dans lequel Émilie du Châtelet souligne les enjeux métaphysiques du principe de conservation:

Mais la seule chose qui m'embarrasse à présent, c'est la liberté, car enfin, je me crois libre et je ne sais si cette quantité de forces toujours la même dans l'univers ne détruit point la liberté. Commencer le mouvement, n'est-ce pas produire dans la nature une force qui n'existait pas? Or, si nous n'avons pas le pouvoir de commencer le mouvement, nous ne sommes point libres ${ }^{21}$.

Dans l'ensemble de lettres qu'Émilie du Châtelet échange avec Maupertuis cette année-là (I738), les enjeux du principe leibnizien de conservation des forces vives sont ainsi clairement posés: I) le choix du principe de conservation suppose des décisions métaphysiques, il n'y a donc pas d'autonomie du principe à l'égard de ses présupposés métaphysiques; 2) ces décisions métaphysiques concernent, pour Leibniz du moins, la possibilité d'utiliser un seul et même principe pour expliquer le mouvement des corps animés et inanimés ${ }^{22}$. C'est dans ce cadre que se pose la question de la liberté humaine. Comme Émilie Du Châtelet l'écrit dans une lettre de mai I 738 adressée au même Maupertuis, le "pouvoir soi-mouvant ", c'est-à-dire la spontanéité de l'actio in se ipsum qui produit la force, pour parler le langage leibnizien ${ }^{23}$, peut être réservé aux seuls êtres animés, là où la force se

21. Lettres de la marquise du Châtelet, éd. Besterman, vol. I, p. 220.

22. Mon interprétation de cet épisode fameux se distingue ici de celle proposée par Matt Jones dans son intervention «From Sociability to Vis viva: Emilie du Châtelet on Social and Natural Order ", lors du colloque «Exploring the Philosophy of Emilie du Châtelet » organisé à l'Université de Columbia en juin 2016. Matt Jones défendait l'idée qu'en I738 Emilie du Châtelet acceptait la mesure mv2 mais en refusait les conséquences métaphysiques et en un sens que la suppression du chapitre sur la liberté lui aurait permis d'adhérer pleinement au principe leibnizien. Il me semble que la démarche d'Émilie peut être interprétée différemment: en tout état de cause, elle adhère au principe de conservation (Voir la lettre à Maupertuis du Io février I738 dans laquelle Émilie indique qu'on peut avoir raison sur les forces vives et se tromper sur les monades: «M. de Leibniz, à la vérité, n'avait guère raison que sur les forces vives »), puis elle élabore la métaphysique qui lui semble compatible avec mv2, ce qui va la conduire à considérer que toutes les substances sont dotées d'action mais pas de perception.

23. Voir Leibniz-De Volder. Correspondance, éd. A.-L. Rey, Paris, Vrin, 20I6, p. 234, Lettre de Leibniz à De Volder du Io novembre I703: "Je reconnais que les Monades sont 
consume, se communique et se conserve à l'occasion du choc des corps inanimés. On pourrait dire que cette proposition de «conciliation» - Émilie du Châtelet écrit en effet dans cette même lettre: "[J]'ai envie de faire un moment le conciliateur comme M. de Mairan ${ }^{24}$ » - est davantage la formulation d'un problème que sa véritable résolution dans la mesure où l'auteure, tentée par la solution wolffienne, en mesure rapidement le coût philosophique: le renoncement à un principe unique d'intelligibilité de toutes les substances, garant de la continuité à l'œuvre dans la nature.

Il peut être utile de relier ces hésitations à la conception qu'Émilie du Châtelet se fait de la liberté. La philosophe avait projeté de faire figurer un chapitre sur la liberté dans les Institutions, elle y a finalement renoncé, mais il est possible d'avoir une idée de sa conception de la liberté en lisant le "chapitre V sur la liberté» attribué à Voltaire et publié par Ira O. Wade ${ }^{25}$. On suit, en effet, ici les conclusions convaincantes de Linda Gardiner Janik qui, pour établir qu'Émilie du Châtelet a en réalité écrit ce chapitre V, s'appuie sur des proximités stylistiques et doctrinales avec les premières versions des Institutions ${ }^{26}$, et tout particulièrement les échos entre ce texte et la première version non publiée du chapitre XXI des Institutions. Que nous apprennent ces textes sur sa conception de la liberté?

Dans le manuscrit autographe de I737, Émilie résume la teneur du $\$ 720$, en marge, par ces mots: «Les forces vives et leur conservation ne sont point contraires à la liberté. » Le paragraphe formule, en termes simples, les conséquences pour la liberté humaine de l'adoption du principe de conservation leibnizien:

actives par soi, dans lesquelles outre la perception qui enveloppe l'action en tout cas, rien ne peut être compris.»

24. Lettre I44-2400 d'Émilie du Châtelet à Maupertuis de mai I738, op. cit., p. 240: "J'ai envie de faire un moment le conciliateur comme M. de Mairan et de dire que Dieu peut avoir établi des lois de mouvement pour le choc des corps inanimés, par lesquelles ils conservent ou communiquent ou consomment dans des effets la force qu'on leur imprime; mais que cela n'empêche point qu'il ne réside dans les êtres animés un pouvoir soi-mouvant, qui est un don du créateur comme l'intelligence, la vie, etc. Car si je suis libre il faut absolument que je puisse commencer le mouvement, et si ma liberté était prouvée il faudrait bien convenir que ma volonté produit de la force, quoique le quomodo me soit caché. La création, qu’il faut bien admettre quand on admet un Dieu, n'est-elle pas dans ce cas-là, et n'y a t-il pas mille choses qu'il nous sera toujours également impossible de nier et de comprendre? »

25. Ira O.Wade, Studies on Voltaire with Some Unpublished papers of Mme Du Châtelet, Princeton, Princeton University Press, I94, p. 92-I08.

26. Voir Linda Gardiner Janik, "Searching for the Metaphysics of Science: the Structure and Composition of Madame du Châtelet's Institutions de physique, I737-I740", Studies on Voltaire and the Eighteenth Century, 20I, I982, p. 85-II3, ici p. 88-89. "My argument for this terminus a quo rests on the identification of the fragmentary "Chapitre V de la liberté" published by Wade [...] and said by him to be Voltaire's work, as having been written in fact by Mme du Châtelet.» 
La conservation d'une quantité égale de force dans notre univers paraît d'abord intéresser notre liberté, mais ce n'est que lorsque l'on confond la quantité du mouvement et la quantité de la force, si nous sommes libres, notre volonté produit sans doute du mouvement, car s'il dépend de moi de me promener ou de rester assis, il est certain qu'en me promenant, je produirai un mouvement que je n'eusse pas produit si j'étais resté sur ma chaise, mais est-il nécessaire pour cela que je produise de la force, non sans doute, je puis être libre dans l'emploi de la quantité de force qui se trouve dans mes nerfs et dans mon sang, sans que pour cela ma volonté puisse créer de la force, ainsi, il me semble que l'on peut mettre au nombre des lois de la nature la conservation toujours égale de la quantité de force vive qui est dans l'univers ${ }^{27}$.

En un mot, le mouvement que je crois produire n'est que la conséquence d'une force que je possède et que je choisis d'exercer à un moment donné, il est donc possible de maintenir la conviction que nous sommes libres, et l'adhésion au principe de conservation des forces vives, à condition de bien distinguer mouvement et force.

Il faut naturellement mettre en regard ce texte avec le fameux "chapitre $\mathrm{V}$ de la liberté ». Dans le chapitre V, quatre objections à la liberté humaine sont examinées. La première concerne le sentiment illusoire de liberté, à laquelle le texte oppose une forme d'introspection: "[M]ais enfin quand il faut s'interroger soi-même, il faut bien avouer, si l'on est de bonne foi, que nous avons une volonté, que nous avons le pouvoir d'agir, de remuer notre corps, d'appliquer notre esprit à certaines pensées, de suspendre nos désirs, etc. ${ }^{28}$ ", ainsi qu'une redéfinition de la liberté fondée sur l'action et non sur la volonté29. Mais les accents leibniziens qui inclinent à penser que le texte est sans doute écrit par un auteur sensible aux thèses leibniziennes se trouvent dans la réponse à la troisième objection, lorsqu'il s'agit de penser le lien entre la liberté humaine et la prescience divine. Cette prescience caractérisée comme une connaissance qui, à ce titre, n'influe pas sur l'existence des choses ${ }^{30}$.

27. Émilie du Châtelet, Institutions de Physique, I737, manuscrit autographe, BNF, Fr, I2265, feuillet numéroté 370 .

28. "Chapitre V de la liberté", in Ira O. Wade, Studies on Voltaire with some unpublished papers of Mme du Châtelet, Princeton, Princeton University Press, I947, p. 96.

29. Op. cit., p. 99: «[C]ar la liberté consiste à agir ou ne pas agir et non pas à vouloir ou ne pas vouloir [...] La volonté, dis-je, ne peut avoir aucune influence sur le pouvoir soi-mouvant en quoi consiste la liberté [...] [L]e pouvoir physique d'agir est donc ce qui fait de l'homme un être libre.»

30. «Car la prescience de Dieu n'est pas la cause de l'existence des choses, mais elle est elle-même fondée sur leur existence. Tout ce qui existe aujourd'hui ne peut pas ne point exister [nécessairement] pendant qu'il existe, et il était hier et de toute éternité aussi constamment vrai que les choses qui existent aujourd'hui devaient exister, qu'il est maintenant certain que ces choses existent. La simple prescience d'une action avant qu'elle soit faite ne diffère en rien de la connaissance qu'on en a après qu'elle est faite. Ainsi la prescience ne change rien à la certitude d'évènement. » 
Si Émilie du Châtelet commence par indiquer que l'idée d'un principe de conservation, c'est-à-dire qu'une même quantité se conserve - qu'il s'agisse du mouvement ou de la force - , lui semble contraire à la liberté définie comme pouvoir de se mouvoir et de ne pas se mouvoir conformément au choix de son propre esprit ${ }^{31}$, elle formule ensuite clairement la hiérarchie qu'elle établit entre l'enjeu de la liberté et le principe de conservation: «[I]l est certain que la question de notre liberté (s'il en est une) nous intéresse infiniment plus que toutes celles que l'on peut faire sur la nature du mouvement et sa conservation, puisque de cette seule question dépend toute la morale. » On peut rappeler que, dès la lettre du Io février I738, à Maupertuis, Émilie du Châtelet se posait les mêmes questions et considérait qu'on pouvait avoir raison sur les forces vives et se tromper sur le plein et les monades ${ }^{32}$.

Mon hypothèse est que faire le lien entre le principe de conservation et la question de la liberté suppose d'utiliser une conception leibnizienne de l'intelligibilité de la substance, c'est-à-dire de choisir une seule et même explication pour tous les types de substances (qu'elles soient brutes ou simples). Les thèses leibniziennes du principe de continuité et de l'uniformité explicative sont bien connues dans cette première moitié du Xviri ${ }^{\mathrm{e}}$ siècle, grâce à la parution des Essais de Théodicée $e^{33}$. Mais comme nous le savons, Wolff établit une différence entre les types de substances, c'est-à-dire une différence dans l'intelligibilité des substances inanimées, dépourvues de perception et des êtres simples qui, eux, sont dotés de perception. Par ailleurs, pour Wolff, la substance est active mais n'est pas perceptive, et c'est précisément ce qu'Émilie du Châtelet récuse dans la conception leibnizienne de la substance: cette perception. Il me semble donc que le chapitre sur la liberté a sans doute disparu pour deux raisons: I) parce qu'Émilie du Châtelet a

31. "Chapitre V de la liberté", in Ira O. Wade, Studies on Voltaire with Some Unpublished Papers of Mme du Châtelet, Princeton, Princeton University Press, I947, p. 92: "Je n'examine point ici si l'opinion qui veut que la quantité de force reste la même dans l'univers est aussi contraire à la liberté que celle d'une égale quantité de mouvement, voy. sur cela chap. VIII.»

32. Lettre d'Émilie du Châtelet à Maupertuis du ıo février I738, op. cit., p. 226: «Le docteur Clarke, dont M. de Mairan a rapporté toutes les raisons dans son mémoire, traite M. de Leibniz avec autant de mépris sur la force des corps que sur le plein et les monades; mais il [a] grand tort à mon gré, car un homme peut être dans l'erreur sur plusieurs chefs et avoir raison dans le reste. M. de Leibniz, à la vérité, n'avait guère raison que sur les forces vives, mais enfin il les a découvertes et c'est avoir deviné un des secrets du créateur. »

33. À titre d'exemple, voici comment Leibniz le formule pour Wolff dans une lettre du 9 novembre 1705 : "D'après moi, selon la grande uniformité de la nature, toutes choses partout dans les grandes et dans les petites, dans les visibles et dans les invisibles se produisent de la même manière, et varient seulement par leur degré de grandeur et de perfection. » Lettre de Leibniz à Wolff du 9 novembre 1705 dans G.W. Leibniz, 1705-1716, Briefwechsel zwischen Leibniz und Christian Wolff, reed. Carl I. Gerhardt, Halle, I 860, Hildesheim/New York, Olms, I97I, lettre VIII, p. 44. 
choisi la réponse wolffienne au problème que lui posaient les principes leibniziens; et 2) parce qu'elle a renoncé à une conception atomiste de la matière au profit de la reconnaissance d'une élasticité essentielle aux corps.

C'est aussi la raison pour laquelle cette objection à l'égard du principe leibnizien de conservation se double d'une autre qui concerne la conception même de la matière qu'elle engage. Pour lever ces objections et clarifier les enjeux du principe de conservation, il faut donc préciser quelle conception de la matière Émilie du Châtelet défend dans les Institutions. En un sens, on pourrait dire que tant qu'elle défend une conception atomiste de la matière ${ }^{34}$ présente dans les lettres contemporaines des questions adressées à Maupertuis en $\mathrm{I} 738$, il ne lui est pas possible d'admettre une force à l'œuvre dans les corps qui s'exprime, se restitue et se conserve grâce à l'élasticité de ces $\operatorname{corps}^{35}$. Mais lorsqu'Émilie comprend le lien entre le choix d'un principe unique d'intelligibilité de la substance et l'adhésion à l'existence d'une force active dans les corps, elle doit développer une conception spécifique de la matière qui lui permette d'articuler ces deux exigences. C'est ainsi qu'elle écrit au $\$ I 5 des Institutions: «Le principe de continuité prouve qu'il n'y a point de corps durs dans l'univers ${ }^{36}$ "

Émilie du Châtelet choisit donc une architecture philosophique: des principes architectoniques qui supposent que la métaphysique fonde la physique $^{37}$. Cette exigence se traduit par le recours au principe de continuité et au principe de simplicité comme conditions d'articulation du rapport entre physique et métaphysique. Les preuves de la pertinence du principe de conservation sont alors l'instrument d'une réévaluation de sa conception de

34. Lettre d'Émilie du Châtelet à Maupertuis du I7 juillet I738, op. cit., p. 262-263: "Quant aux atomes, s'ils sont matière, comme ils le sont sans doute puisqu'un composé ne peut être fait de parties essentiellement différentes de lui-même, puisque, dis-je, ils sont matière, il faut bien qu'ils aient une figure. Je sais bien que nous ne pouvons la voir, mais nous devons et nous pouvons la supposer, et je crois qu'il est assez naturel de la supposer sphérique.»

35. Lettre d'Émilie du Châtelet à Maupertuis du Io février I738, op. cit., p. 226: "Je vous avoue qu'il me reste une grande peine d'esprit sur ce que vous me dites, que si l'on prend pour forces les forces vives, la même quantité s'en conservera toujours dans l'univers. Cela serait plus digne de l'éternel géomètre, je l'avoue, mais comment cette façon d'estimer la force des corps empêcherait-elle que le mouvement ne se perdît par les frottements, que les créatures libres ne le commençassent, que le mouvement produit par deux mouvements différents ne soit plus grand quand ces deux mouvements conspireront ensemble, que lorsqu'ils seront dans des lignes perpendiculaires l'un à l'autre, etc. ? Il y a peut-être bien de la témérité à moi à vous supplier de me dire comment il s'ensuivrait qu'il y aurait dans l'univers la même quantité de force, si la force d'un corps en mouvement est le produit de sa masse par le carré de sa vitesse. J'imagine qu'il faudra peut-être distinguer entre force et mouvement, mais cette distinction m'embarrasse extrêmement, et puisque vous avez jeté ce doute dans mon esprit j'espère que vous l'éclaircirez» (c'est moi qui souligne).

36. Émilie du Châtelet, Institutions de physique, chapitre I, \$I 5, Paris, Prault, I740, p. 34 .

37. Il n’y a qu'à rappeler la formule citée plus haut: «mais comme je suis persuadée que la physique ne peut se passer de la métaphysique sur laquelle elle est fondée». 
la matière, qui se conclut par un renoncement à l'atomisme et par l'adhésion à l'idée leibnizienne d'une force interne présente dans les corps.

Quel est le dispositif argumentatif qui rend possible l'adoption de cette nouvelle conception de la matière?

\section{Les enjeux de la définition de la matière}

Dans les lettres importantes de 1738 adressées à Maupertuis, les enjeux métaphysiques du principe de conservation sont explicités. Si l'on veut résumer les arguments formulés par Émilie du Châtelet, on peut dire, tout d'abord, qu'ayant admis le principe de conservation des forces vives, l'existence de corps parfaitement durs est posée comme une objection à l'élasticité des corps sur laquelle repose cette même estime. L'objection interroge la restitution des forces après le choc et plus fondamentalement leur conservation dans un monde où l'on n'admet que les atomes et le vide ${ }^{38}$. Autrement dit, une fois le principe de conservation leibnizien admis - et l'on peut évidemment faire référence ici au rôle que les expériences menées par les physiciens hollandais (Willelm's Gravesande) ${ }^{39}$ et italien (Giovanni Poleni),

38. Voir Lettre à Maupertuis du 9 mai I738, op. cit., p. 239: "Je vois (autant que je peux voir) qu'il est certain que la force ou l'effet de la force des corps est le produit de la masse par le carré de la vitesse, et que la quantité de force d'un corps et la quantité de mouvement de ce corps sont deux choses très différentes. Cela étant accordé par ceux qui combattent les forces vives, je ne vois pas trop ce qu'ils combattent et j'ai bien peur que cela ne ressemble aux moulins à vent [...]. Mais je vois qu'il y a plus de difficulté que je ne croyais dans le détail, car je vous prie de me dire, et M. Newton l'a demandé avant moi, ce que deviendrait la force de deux corps durs qui se choqueraient dans le vide. Car alors il ne peut y avoir de dispersion de mouvement entre leurs parties ou bien entre les corps voisins. Je sais qu'on ne connaît point jusqu'à présent de corps parfaitement dur, mais ce n'est pas, je crois, une démonstration qu'il n'y en ait point, et je ne sais même s'il n'est pas nécessaire d'en admettre dans la nature quoique nous n'ayons pas d'organes ni d'instruments assez fins pour les discerner. Or dès qu'il peut exister des corps parfaitement durs, et qu'il est même très vraisemblable que les premiers corps de la matière le sont, il est permis de considérer ce qui arriverait à de tels corps qui se choqueraient dans le vide. Or certainement ils ne rejailliraient pas, que deviendrait donc leur force? Car il n'y a point là d'enfoncement, point de ressort prêt à rendre la force qui le tient tendu au corps qui la lui a donnée.»

39. Indiquons pour mémoire que, dans une lettre à Prault de juillet I738 (op. cit., p. 259), Du Châtelet mentionne quelques livres déjà présents dans sa bibliothèque et en commande d'autres. "Je vous prie de me mander si l'Académie des sciences, que vous trouvez à vendre, est toute reliée et à combien cela montera, et s'il faudrait le payer tout à l'heure. Je veux encore les Transactions philosophiques, la République des lettres jusqu'à la mort de Bayle, et tous les livres de physique que vous trouverez dans votre chemin. À mesure que je m'en souviendrai, je les mettrai sur une carte et vous en enverrai la liste. J'ai l'Optique de Newton 3, Rohaut commenté par Clarke 4, Whiston 5 , La Figure de la terre, Figure des astres, Musschenbroeck Physique 's, Gravesande Physique, Recueil des lettres de Leibniz et de Clarke, les Entretiens physiques du père Regnault pour ce qu'ils valent, Euclide, Pardies, Malezieu, l'Application de l'algèbre à la géométrie de Guisnée, les Sections coniques de M. de L'Hôpital, les Mathématiques universelles et les Euvres de Descartes. Voilà à peu près tout. Je vous prie de me chercher 
citées dans les Institutions ${ }^{40}$, a eu sur la conversion d'Émilie du Châtelet quelle est la conception de la matière qui permet de le fonder? Dans cette quête, Émilie s'appuie à la fois sur le questionnement newtonien ( «M. Newton l'a demandé avant moi, ce que deviendrait la force de deux corps durs qui se choqueraient dans le vide») et sur les "solutions " élaborées par Maupertuis. En effet, "prendre métaphysiquement les effets pour les forces ${ }^{41}$ » permet de concevoir ce que Leibniz désignait comme un effet formel, essentiel au mouvement, qui résulte d'une action surmontant l'inertie naturelle des corps, et, corrélativement, permet de poser la question des usages légitimes de la métaphysique. Ces lettres de 1738 confirment donc que c'est sur le plan métaphysique - les échanges portent sur la nature de la matière que se situe la discussion sur la pertinence du principe de conservation des forces vives. Elles nous apprennent également que ce sont des raisons métaphysiques qui ont convaincu Maupertuis de la validité de la loi d'attraction. La pique que Du Châtelet adresse à Maupertuis est particulièrement significative de cela:

Je vous croyais réconcilié avec elle [la métaphysique] depuis que vous aviez décidé pour la loi d'attraction en raison inverse du carré des distances en faveur de l'une raison métaphysique, mais je vois bien que vous n'en voulez que lorsqu'elle justifie les lois établies par le créateur et découvertes par Newton ${ }^{42}$.

Les échanges avec Maupertuis en I738 introduisent donc une justification de la «raison métaphysique de préférence pour la loi d'attraction" (lettre du 2I juin I738), c'est-à-dire que le motif de la raison métaphysique passe de la justification du principe leibnizien de conservation à celle de la loi newtonienne d'attraction. Ce sont pour des raisons métaphysiques (!) que Maupertuis adhère à la philosophie de Newton, et on note ainsi qu'il y a bien une réelle tradition d'articulation de la métaphysique et de l'empirisme qui se précise dans la justification métaphysique de l'adhésion à Newton.

les Principia mathematica de M. Newton, d'une belle édition, et de me les faire relier en cuir de roussi, dorés sur tranches, le plus tôt que vous pourrez; je vous en serai obligée.»

40. Voir Institutions, chapitre XXI, $\mathbb{S} 584$ : «Mr de s'Gravesande a imaginé une expérience qui confirme merveilleusement cette théorie.»

41. "Votre idée de prendre métaphysiquement les effets pour les forces me paraît admirable, car je ne sais si elle ne pourrait point fournir une réponse à cette objection qui m'a toujours arrêtée et qu'à mon gré M. de Bernoulli a trop méprisée. Je crois donc, s'il m'est permis d'avoir une opinion sur cela, que la force de ces corps se consommerait réellement dans les efforts qu'ils feraient pour surmonter réciproquement leur impénétrabilité et leur force d'inertie, et que cet effet qu'ils auraient produit l'un sur l'autre en surmontant la force que tout corps en mouvement a pour persévérer à se mouvoir, cet effet, dis-je, représente métaphysiquement la force qui la produit, et ce serait bien alors que la métaphysique serait contente " (même lettre du 9 mai I 738 ).

42. Voir la même lettre du 9 mai 1738 adressée à Maupertuis. 
En 1738 , les choix philosophiques relatifs à l'architecture de la pensée d'Émilie du Châtelet sont donc faits, il lui reste à formuler une conception de la matière qui lui permette de les rendre cohérents: ce sera le travail des Institutions $^{43}$.

Les premiers chapitres des Institutions de Physique sont souvent présentés comme un moment important de l'introduction des idées leibniziennes en France. Les chapitres VII et VIII respectivement consacrés aux "éléments de la matière » et à «la nature des corps ${ }^{44}$ ne font pas exception à la règle. De l'aveu d'Émilie elle-même, c'est bien l'intention qui préside, du moins, à la rédaction du chapitre VII, comme elle l'écrit au \$I I 9 des Institutions:

Peu de gens en France connaissent autre chose de cette opinion de $\mathrm{Mr}$ de Leibniz que le mot de Monades. Les Livres du célèbre Wolff dans lesquels il explique avec tant de clarté et d'éloquence le système de $\mathrm{Mr}$ de Leibniz, qui a pris entre ses mains une forme toute nouvelle, ne sont point encore traduits dans notre langue, je vais donc tâcher de vous faire comprendre les idées de ces deux grands Philosophes sur l'origine de la Matière ${ }^{45}$.

Une première question se pose aussitôt: s'agit-il vraiment d'idées leibniziennes ? ${ }^{46}$

Il est, en effet, particulièrement intéressant de noter que, dans le cadre usuel de la "clarification du système », et contre le motif galvaudé d'un prétendu «système leibnizo-wolffien", Émilie du Châtelet indique qu'elle reconnaît une différence claire entre les deux pensées de Leibniz et de Wolff. Et l'un des lieux majeurs sur lesquels s'exerce cette différence est, de manière centrale, la conception de la substance. Émilie du Châtelet ne s'y est pas trompée puisque, si elle commence par mentionner les monades leibniziennes, elle les abandonne vite au profit d'un usage quasi-exclusif des «êtres simples ", qui est le terme utilisé par Wolff, et qui revêt pour lui une signification bien distincte.

43. Voir $\$ 557$, chapitre XXI, «De la force des corps»: «Vous avez vu dans le chapitre premier, que le principe de continuité, fondé sur celui de raison suffisante ne souffre point de saut dans la nature, et qu'un corps ne saurait passer d'un état à un autre, sans passer par tous les degrés qui sont entre deux.»

44. Rappelons, pour mémoire, que les chapitres que Wolff consacre à ces questions dans la Cosmologia generalis s'intitulent respectivement «De Essentia et Natura corporum» (section II, chapitre I) et «De elementis corporum " (section II, chapitre II).

45. C'est moi qui souligne.

46. Lettre 28I à Frédéric, août I740: "J'ai le dessein de donner en français une philosophie entière dans le goût de celle de $\mathrm{M}$. Wolff, mais avec une sauce française; je tâcherait de faire la sauce courte. Il me semble qu'un tel ouvrage nous manqué. Ceux de M. Wolff rebuteraient la légèreté française par leur forme seule; mais je suis persuadée que mes compatriotes goûteront cette façon précise et sévère de raisonner, quand on aura soin de ne les point effrayer par les mots de lemmes, de théorèmes et de démonstrations, qui nous semblent hors de leur sphère quand on les emploie hors de la géométrie.» 
Dans ce cadre, la correspondance entre Leibniz et Wolff donne accès à la reprise par Wolff des thèmes leibniziens ou au contraire aux divergences assumées. On peut ensuite apprécier ces divergences dans les Principia dynamica (I728) et la Cosmologia generalis, en particulier aux chapitres II et IV de la section II consacrée à la définition de la notion de $\operatorname{corps}^{47}$. Si Wolff est fidèle à l'estimation du principe de conservation leibnizien, dans certains paragraphes de l'Ontologia ${ }^{48}$ et d'autres paragraphes de la Cosmologia generalis, on relève deux divergences fondamentales qui l'opposent à Leibniz: sur la définition de la substance et sur la conception du rapport entre physique et métaphysique. Et cela est d'autant plus important, qu'il est désormais établi qu'Émilie du Châtelet a eu accès, avant l'arrivée de Samuel Koenig à Cirey, à un certain nombre de textes ${ }^{49}$ qui lui permettent de faire une claire différence entre les pensées de Leibniz et de Wolff, ce dont témoignent d'ailleurs les Institutions.

Mon hypothèse d'interprétation est que c'est ce double refus qu'Émilie du Châtelet va travailler en partant des principes leibniziens. Cela la conduira à élaborer une philosophie naturelle originale et inventive.

Émilie du Châtelet a les moyens de mesurer la différence entre ces deux philosophies, et elle va utiliser cette compréhension de la différence comme un moyen de résoudre les problèmes propres à la philosophie de Leibniz. Elle est confrontée à deux problèmes internes à la philosophie de Leibniz: I) en effet, bien qu'elle introduise effectivement le terme de "monades" au \I I9 pour rendre raison de l'étendue de la matière, elle va par la suite n'utiliser que le terme wolffien d' "être simple». Du Châtelet privilégie par ailleurs l'activité de la substance, issue de la dynamique, à sa dimension perceptive. 2) Mais elle a quelques réticences à concevoir que toutes les substances puissent percevoir. Elle écrit ainsi, au \I32: "C’est encore un des sentiments de M. Leibniz qui a le plus besoin d'être éclairci, que cette représentation de l'Univers et de tous ses changements, dans laquelle il prétend que l'essence de notre âme consiste. » Elle va néanmoins utiliser le motif de la perception distincte et confuse pour donner un statut au phénomène.

C'est précisément l'articulation de ces deux points qui va faire ce que je considère comme la singularité de sa démarche: elle va construire un lien explicite entre l'ontologie et la gnoséologie qui lui permet de résoudre les problèmes que lui pose la philosophie de Leibniz et qui sont résumés dans cette phrase: «Ce qui dans les phénomènes est montré de manière étendue

47. Christian Wolff, Cosmologia generalis, section II «De notione corporum, ex quibus mundus componitur ", I73 I, GW4, Olms I964, p. 228-392.

48. Christian Wolff, Philosophia prima sive Ontologia, I730, Olms. Pour le principe de raison suffisante, voir part. I, sec. I, chap. II, $\$ 56-78$, par exemple le scholie du $\$ 75$, et surtout pour la définition de la substance, part. II, sect. II, chap. I-II.

49. Voir «Lettre à Johann Bernoulli II» de juin I 740 dans laquelle elle indique ses lectures leibniziennes pour montrer en quoi l'accusation de plagiat proférée par Samuel Koenig est infondée. 
et mécaniquement dans les Monades est concentré et doté d'un principe de vie $^{50}$.»

Les deux chapitres VII et VIII des Institutions correspondent respectivement à un mouvement de descente de l'être simple aux corps en mouvement qui porte sur leur statut ontologique, puis de remontée des corps en mouvement à ce que nous pouvons en connaître, c'est la dimension gnoséologique qui est alors visée. On pourrait croire qu'il y a une forme de tension entre ces deux mouvements mais l'habileté d'Émilie du Châtelet est épistémologique: du principe aux choses on comprend selon la raison des phénomènes à leurs justifications, on délimite le statut des expériences dans la connaissance.

Dans un premier temps, Émilie utilise deux principes architectoniques posés dans le premier chapitre pour construire sa conception de la matière. Ainsi, le principe des indiscernables qui, au $\$ I 2$, indiquait l'impossibilité qu'il y ait deux êtres semblables dans la nature a imposé l'idée que la différence entre les êtres doit nécessairement être interne. Du Châtelet convoque ensuite le principe de raison suffisante pour justifier la différence de nature entre l'explanandum et l'explanans, de telle sorte que, puisque l'être simple est saisi par l'entendement et non par l'imagination, nous ne pouvons pas en avoir d'image, on ne peut donc prouver empiriquement la réalité des êtres simples. Mais par conséquent, en vertu du principe de raison suffisante, l'être simple ne peut être un atome qui, lui, est étendu, ne peut pas non plus être un être composé, et donc doit être quelque chose d'inétendu ${ }^{51}$.

50. «Lettre de Leibniz à Wolff» du 9 juillet I7II, dans Leibniz (G. W.), I705-I7I6, Briefwechsel zwischen Leibniz und Christian Wolff, rééd. Carl I. Gerhardt, Halle, I860, ici Hildesheim, New York, Olms, I97I, ici I97I, lettre n 67, voir en part. p. I38-I39.

51. Voir $\$ \mathrm{I} 20$ "Tous les corps sont étendus en longueur, largeur et profondeur; or, comme rien n'existe sans une raison suffisante, il faut que cette étendue ait sa raison suffisante par laquelle on puisse comprendre, comment, et pourquoi elle est possible; car de dire, qu'il y a de l'étendue, parce qu'il y a de petites parties étendues, ce n'est rien dire, puisque l'on fera la même question sur ces petites parties que sur le tout, et que l'on demandera la raison suffisante de leur étendue: or comme la raison suffisante oblige d'alléguer quelque chose qui ne soit pas la même que celle dont on demande la raison, puisque sans cela on ne donne point de raison suffisante, et que la question demeure toujours la même, si l'on veut satisfaire à ce principe sur l'origine de l'étendue, il faut en venir enfin à quelque chose de non-étendu, et qui n'ait point de parties, pour rendre raison de ce qui est étendu et qui a des parties: or un Etre non-étendu et sans parties, est un Etre simple. Donc les composés, les Etres étendus existent, parce qu'il y a des Etres simples. Il faut avouer que cette conclusion étonne l'imagination, les êtres simples ne sont point de son ressort, on ne peut se les représenter par des images et l'entendement seul peut les concevoir... car lorsqu'on dit qu'il y a des corps étendus parce qu'il y a des atomes, c'est comme si l'on disait, il y a de l'étendue parce qu'il y a de l'étendue; ce qui est en effet ne rien dire du tout. On ne peut donc trouver la raison suffisante d'un Etre étendu et composé que dans des Etres simples et non étendus, de même que la raison suffisante d'un nombre composé ne peut se trouver que dans un nombre non composé, c'est-à-dire dans l'unité. Il faut donc convenir, concluent ces Philosophes [les Leibniziens] qu'il y a des Etres simples puisqu'il y a des Etres composés. » 
En imposant et en justifiant une entité immatérielle non perceptible et non susceptible d'être saisie par l'imagination, au fondement de l'explication de la matière et des êtres composés, Émilie du Châtelet, paradoxalement, renverse la difficulté argumentative classiquement rencontrée pour fonder cette thèse. En effet, elle affirme que ne pas voir les êtres simples ou ne pas pouvoir les imaginer n'est pas une faiblesse ou une limite de cette conception, c'est précisément le moyen de prouver le statut des êtres simples. En suivant la suite de l'argumentation, évaluons la force de cet argument.

Les êtres simples sont l'origine des êtres composés ( $\$$ I 25 ), ils sont leur raison suffisante. Il faut donc trouver un principe à l'origine du mouvement perpétuel qui anime les êtres composés, autrement dit un principe d'action qu'elle identifie dans la force: «le principe qui rend compte de l'actualité d'une action, c'est la force» (\$I 26) qui s'exerce si aucune résistance ne l'empêche d'agir.

C’est ce qui permet à Émilie de conclure au $\$ I 28 que les êtres simples sont les véritables substances qui sont actives. Et au I $_{3}$ I : "[L]es raisons primitives de ce qui arrive dans les corps se trouvent dans les éléments dont ils sont composés ", et c'est dans ce cadre qu'elle introduit les variations entre distinction et confusion.

On pourrait jusqu'à maintenant considérer qu'Émilie du Châtelet, sans privilégier l'usage du terme de monade, attribue les caractéristiques de la substance simple leibnizienne aux êtres simples mais conserve en même temps l'une des difficultés centrales de la philosophie leibnizienne: comment les êtres composés peuvent-ils être composés d'êtres simples? Ou pour le dire autrement comment la matière peut-elle être composée d'entités immatérielles? C'est le problème auquel Du Châtelet est à son tour confrontée et auquel elle va essayer de répondre en travaillant dans un deuxième temps, la dimension phénoménale de l'étendue.

\section{L'espace de la phénoménalité}

Ainsi, si l'étendue nous apparaît comme une substance (\$I36), ce ne peut être avec les yeux de l'entendement, car elle n'est qu'un phénomène, et cette illusion de réalité vient de la confusion de la perception et des idées qui produit le phénomène de l'étendue ${ }^{52}$. Ce qui, par conséquent, confirme l'idée que les monades ne peuvent jamais être accessibles par l'expérience ( $\$$ I36). En revanche, nous avons accès, par l'expérience, à l'activité des corps, c'est-

52. Institutions de Physique, $\mathbb{1}$ 35: «[C]ette même confusion fait que le Phénomène de l'étendue résulte pour nous de l'assemblage des Etres simples et de leurs différences internes, mais comme il est impossible que nous nous représentions l'état interne de tous les Etres simples, duquel, cependant, le Phénomène de l'étendue dépend, toute perception des réalités doit nous échapper par notre nature et il ne nous reste des idées confuses que nous avons de chacun de ces Etres simples, qu'une idée de plusieurs choses coexistantes, et liées ensemble, sans que nous sachions distinctement comment elles sont liées, et c'est cette idée confuse qui fait naître le Phénomène de l'étendue.» 
à-dire à la trace ou preuve du principe actif de l'être simple $\left(\mathbb{S}_{\mathrm{I}} 38\right)$. On voit bien ici comment l'activité constitue une sorte d'interface entre la manifestation substantielle et l'expérience perceptive.

C'est l'occasion pour Émilie du Châtelet de signaler au \I 5 I que l'observation de la matière et de la force agissante qu'elle assimile à des phénomènes nous incite à remonter à quelque chose qui les explique, c'est-à-dire les Éléments ${ }^{53}$. La confusion est constitutive de la phénoménalité et est liée au degré d'imperfection de nos organes. Si notre vue était plus distincte, les phénomènes seraient des êtres simples. Cela conduit à élaborer une définition des phénomènes qu'on trouve au $\mathbb{S I}_{54}$ : «des images ou des apparences qui naissent par la confusion de plusieurs réalités ${ }^{54} »$.

Le champ phénoménal est certes la marque de notre limite et de notre confusion perceptive, mais il est aussi, et en un sens malgré tout, le lieu de présence de la substantialité, et c'est ce qui importe ici. Il peut, à cet égard, être précieux de se référer à la définition que Wolff propose du phénomène ${ }^{55}$ dans la Cosmologia generalis, précisément comme ce qui est perçu confusément par les sens.

Or ce passage de l'ontologie à la gnoséologie me semble pouvoir être rapproché de la distinction qu'opère Wolff, dans le Discursus praeliminaris, au $\$ 94$, entre deux ordres: la méthode démonstrative et la méthode de l'étude. En effet, en prenant l'exemple de la physique, Wolff indique bien que si l'on procède selon l'ordre démonstratif en physique, il faut alors avoir recours aux principes de la physique présents dans la cosmologie et dans l'ontologie. Il conclut sur ce point en rappelant que ces disciplines sont ellesmêmes des parties de la Métaphysique. Il peut être opportun de rapprocher ce passage du \$ro9 dans lequel Wolff développe l'idée selon laquelle il faut dériver de la physique expérimentale les principes de la science de la nature. De sorte que se fait jour dans le texte wolffien un double régime de justification: si l'on suit la «méthode de l'étude » (\$IO7 du Discursus praeliminaris),

53. Op. cit., \$ I 5 I : « [M] ais comme d'un côté les Phénomènes montrent la substantialité de la force active, de même que celle de la matière, et que de l'autre, il y a des difficultés insurmontables qui s'y opposent, on en doit conclure que ni la matière, ni la force active ne sont de véritables substances, mais qu'il faut remonter plus haut et chercher leur source dans quelque chose d'antérieur, d'où l'on puisse montrer pourquoi la force active et la matière doivent paraître des substances, et des substances différentes, cette recherche nous conduira aux Eléments qui sont la source commune de l'une et de l'autre. »

54. Cette conceptualisation de la phénoménalité indexée à notre perception bornée avait été préparée dans les paragraphes précédents, voir par exemple $\$ 152$ : "La matière et la force active qui nous paraissent des substances, n'en sont pas réellement; de même que l'on a vu ( I 34 ) que l'étendue n'est pas une substance, mais un agrégat, un composé de substances: il en est de même de la force active et de la force passive, ce ne sont que des Phénomènes qui résultent de la confusion qui règne dans nos organes et dans nos perceptions. »

55. Ch. Wolff, Cosmologia generalis, section II, chapitre III «De Orto corporum ex elementis ", en particulier, $\mathbb{\$} 225$ : "Phaenomenon dicitur, quicquid sensui obvium confuse percipitur". 
c'est de l'expérience qu'il est possible de tirer les principes de la physique, à rebours, si l'on suit la «méthode démonstrative » $\$ 95$ du même texte), c'est de la métaphysique qu'il convient de tirer ces mêmes principes.

Le rapprochement met en évidence la coexistence chez Wolff de deux modes d'accès à une même réalité, déclinée selon des niveaux d'intelligibilité différenciés. À notre sens, l'enjeu de cette coexistence, sous couvert de dissocier logique cognitive et logique démonstrative, est de proposer l'articulation de deux visages classiquement opposés de la philosophie naturelle ${ }^{56}$. Pour Wolff, la méthode de l'étude sert à rendre compte des raisons dernières, c'est-à-dire de la réalité particulière des choses, ce que la démonstration présente dans l'ontologie et la cosmologie générale est incapable de fournir ${ }^{57}$.

Il n'y a évidemment aucune certitude sur le fait qu'Émilie du Châtelet ait eu accès à ce texte, directement ou par le biais de Koenig, mais il est néanmoins fort possible que ce motif, finalement assez commun, d'une distinction entre une logique de diffusion et une logique proprement démonstrative ait pu être transmis par Koenig à Émilie du Châtelet.

Pour expliquer que les monades sont des substances et que nous n'avons accès à la substantialité que par les corps, Leibniz utilisait l'ambivalence de l'action à la fois essence de la substance et objet du principe de conservation des corps en mouvement. Ce que fait Émilie du Châtelet est différent: elle reprend la catégorie wolffienne de phénomène substantié, mais dans le cadre de la pensée leibnizienne, c'est-à-dire dans le cadre d'une pensée qui ne fait pas une différence de nature entre les êtres simples et les monades brutes. Mais elle lui confère une signification nouvelle.

Au $\$ I 56, elle écrit:

Les trois propriétés [étendue, force passive, force active] qui font l'essence du corps sont donc des phénomènes, mais on peut dire que ce sont des Phénomènes substantiés, comme les appelle $\mathrm{M}$. Wolff, càd des Phénomènes qui nous paraissent des substances mais qui n'en sont cependant pas; car il n'y a de véritables substances que les Etres simples, or comme nous avons vu dans le chapitre précédent que les Eléments doivent contenir l'origine de tout ce qui se trouve dans les Corps qui en sont des composés, puisqu'il se trouve de l'action et de la résistance dans les Corps, on en doit conclure que les Etres simples ont un principe actif, par lequel on peut comprendre pourquoi les composés agissent, et un principe passif, d'où les passions, ou la faculté de pâtir des composés, résulte.

Émilie du Châtelet utilise donc cette notion de "phénomène substantié » pour désigner un phénomène qui nous paraît une substance mais qui

56. Nous avons développé ce point dans «Leibniz et Newton dans Wolff: un précurseur pour les Lumières européennes?" dans La pensée encyclopédique de Ch. Wolff. Autour du Discursus praeliminaris, Lumières, $\mathrm{n}^{\circ} \mathrm{I} 2,2008$, Bordeaux, p. I 25 -I 37.

57. Je remercie le critique anonyme de l'article de m'avoir incitée à mentionner ce point. 
n'en est pas une, car les véritables substances sont des êtres simples. Par le recours à cette expression, elle indique, après Wolff, ce qu'est réellement l'étendue, à savoir un phénomène et la manière dont il nous apparaît, à savoir comme une substance. Mais chez Wolff, dans un texte de I73 I intitulé «De notione corporis ${ }^{58}$ ", le recours à cette expression s'inscrit dans le souci d'une distinction conceptuelle qu'il introduit entre ces phénomènes substantiés et les "phénomènes fondamentaux", respectivement phaenomena substantiata et phaenomena fundamentalia. L'enjeu est de pouvoir distinguer les phénomènes conçus par accident qui n'ont pas en eux-mêmes leur raison d'être, des phénomènes dits substantiés, en ce qu'ils ont en euxmêmes leur raison suffisante et qui, pour cette raison, peuvent être conçus «à la manière des substances".

Elle utilise cette expression dans un sens un peu différent de celui de Wolff: lui cherchait à distinguer entre les entités possédant en elles-mêmes leur raison d'être et celles subordonnées aux premières. À cet égard, la position d'Émilie du Châtelet est double dans la mesure où tout à la fois elle affirme, par le recours à cette expression, la distinction entre ce qui nous apparaît et ce qui est réellement: l'étendue n'est qu'un phénomène mais elle nous apparaît comme une substance, et dans le même temps, elle explique en quelque sorte la confusion possible de ceux qui «ont pris l'étendue pour une substance ", à savoir les cartésiens. Cette confusion s'explique, selon la leçon leibnizienne, par un défaut de perception. Par le recours à cette expression de "phénomène substantié ", elle rend bien compte de sa propre position à l'égard de la matière et de la force: une position éminemment leibnizienne qui considère que seuls les êtres simples sont dotés d'une substantialité dont témoigne l'activité présente dans les corps.

Ces deux éléments, une activité, plutôt qu'une perception, et un être simple, plutôt qu'une monade, nous paraissent révélateurs de son rapport singulier à la notion de substance.

Il semble en effet qu'Émilie du Châtelet ait été, un temps, tentée par la réforme métaphysique qu'opère Ch. Wolff: ce dernier, contrairement à Leibniz, qui considérait toute substance comme perceptive, préfère insister sur la distinction entre les types de substances. Il fait le départ entre les éléments des corps, non dotés de perception, que Leibniz appellerait des monades brutes, et les monades proprement dites qui, elles, sont perceptives. En réintroduisant deux types de substances régis par des régimes distincts, Wolff suit la position cartésienne. À l'inverse, en considérant qu'il est

58. Christian Wolff, Horae subsecivae Marburgenses édité par Jean Ecole, Gesammelte Werke, II, Abteilung, Lateinische Schriften; 34, III, (I73 I, I édition I735) Hildesheim; Zurich; New York: G. Olms, I983. "De notione corporis", Pars altera, p. I23-I4I ; on pense ici au début du $\$ 3$, p. I 28: «Haec tamen phaenomena ab aliis differunt. Etenim cum phaenomena alia, veluti colores et ceterae qualitates sensibiles, per modum accidentium concipiantur, quae subjecto inhaerent, ex adverso materia et vis motrix, quemadmodum modo vidimus $(\$ 2)$, per modum substantiarum concipiendae sunt.» 
possible de concevoir un principe d'intelligibilité unique pour toutes les formes de substances, Leibniz proposait une nouvelle appréhension fondée sur le système de l'harmonie préétablie.

On peut donc récapituler ce point en disant qu'Émilie du Châtelet retravaille les acquis de la métaphysique leibnizienne grâce à la connaissance des textes de Wolff: si la difficulté de Wolff à considérer la perception comme l'apanage de toutes les formes de substance est bien prise en charge par Émilie, cela ne la conduit pas à faire retour à la dualité des substances wolffienne, mais à s'en tenir à une essence active de la substance qui lui permet de penser autrement que Wolff la phénoménalité, par le biais du phénomène substantié. Elle pense en effet la phénoménalité comme le territoire sur lequel l'intelligibilité de l'action des monades est possible. Mais les derniers paragraphes du chapitre VIII indiquent autre chose. Pour rendre raison des phénomènes et de la nature des corps, il faut certes tâcher d'identifier les principes qui permettent d'expliquer pourquoi les changements arrivent dans les corps (la puissance active et la force) et, en régime leibnizien, c'est bien la présence de la substantialité dans le phénomène, quoique confusément, qui rend cela pensable. Mais Émilie du Châtelet ajoute autre chose, dans les tout derniers paragraphes, pour trouver "la raison des effets", il n'est pas toujours nécessaire de remonter à la raison première des choses, il peut donc suffire d'utiliser un phénomène pour rendre raison d'un autre ${ }^{59}$.

À un premier niveau, on pourrait interpréter ce chapitre VIII comme la reprise du dispositif que l'on peut lire dans le $\$ 22$ du Discours de méta$p_{\text {physique }}^{60}$ : il y a une explication mécanique des phénomènes dont on doit rendre raison par la remontée aux causes finales.

Mais le geste essentiel d'Émilie du Châtelet est d'inscrire l'hypothèse centrale de Newton - l'attraction - dans le cadre de cette explication mécanique. De telle sorte qu'en faisant cela, Du Châtelet construit un dispositif dans lequel il y a d'une part une raison première de tous les phénomènes qui s'explique par la présence confuse de la substantialité dans les phénomènes, et d'autre part, une explication mécanique possible des phénomènes, et centralement, du phénomène de l'attraction, qui ne suppose pas de remonter à une raison première mais qui n'en est pas moins, "concevable

59. \I63: «Quand on dit qu'il faut tâcher de rendre raison de tous les effets naturels par la matière et le mouvement, cela ne veut pas dire que l'on soit obligé de trouver cette raison pour tous les Phénomènes, ni de remonter jusqu'à la raison première des choses; la faible portée de notre esprit et l'état présent des Sciences ne le permettent pas: mais on peut s'arrêter à des qualités Physiques, et se servir d'un Phénomène ou de plusieurs, dont on ne connaît point encore les raisons mécaniques (quoiqu'ils en aient) pour rendre raison d'un autre Phénomène qui en dépend.»

60. Dont le titre est: "Conciliation des deux voies par les finales et par les efficientes, pour satisfaire tant à ceux qui expliquent la nature mécaniquement qu'à ceux qui ont recours à des natures incorporelles ", G.W. Leibniz, Discours de métaphysique. 
distinctement et explicable intelligiblement ${ }^{61}$ », à condition de ne pas prendre l'attraction "pour une propriété inhérente de la matière ». Par là, elle délivre aussi ce qui lui semble être l'interprétation correcte du texte newtonien.

Elle arrime ainsi doublement la physique à la métaphysique: une première fois en posant la question de la cohérence entre principe de conservation et conception de la liberté, ce qui la conduit à expliciter ce qu'elle entend par liberté, et une deuxième fois en affirmant que le fondement substantiel des phénomènes ne doit pas nous empêcher d'acquérir une connaissance phénoménale certaine, ce qui lui permet d'inscrire la physique de Newton dans le dispositif métaphysique leibnizien.

\section{Conclusion}

À lire Émilie du Châtelet, on saisit à quel point l'introduction des idées leibniziennes et wolffiennes en France dans les années I 730 modifie significativement la compréhension que l'on peut avoir de la philosophie naturelle de la première moitié du XVIII ${ }^{e}$ siècle bien souvent lue au prisme des oppositions rassurantes (cartésiens contre newtoniens, métaphysiciens contre tenants de l'expérimental philosophy, etc.). La situation philosophique d'Émilie du Châtelet ne peut se réduire ni à une labellisation (leibnizienne ou wolffienne plutôt que newtonienne), ni à une forme de syncrétisme ou encore d'éclectisme conciliatoire ${ }^{62}$ pour reprendre la belle formule de Christia Mercer à propos de Leibniz. Il me semble en effet qu'Émilie du Châtelet reconfigure le champ de la phénoménalité et sa fonction: un lieu d'intelligibilité de l'activité de la substance, et circonscrit dans le même temps un nouvel espace épistémique qui correspond à ce que nous pouvons savoir distinctement. Du Châtelet s'affirme donc comme une philosophe à part entière, car elle déplace et reconfigure le périmètre d'un problème philosophique et contribue significativement à faire exister en France une tradition philosophique qui articule métaphysique et philosophie expérimentale.

Ainsi, la Minerve vient, effectivement, de faire sa physique!

61. Pour paraphraser les derniers mots du chapitre VIII, $\$$ I 64 : "C’est ainsi qu'on peut et qu'on doit se server de l'attraction comme d'une qualité Physique, dont la cause mécanique est inconnue, pour rendre raison d'autres Phénomènes qui en résultent. Ainsi, on peut assurer, par exemple, que le Soleil attire les Planètes et d'autres matières qui les environnent, puisque les Phénomènes le démontrent, pourvu qu'on ne fasse pas de cette attraction une propriété inhérente de la matière et qu'on ne détourne pas les Philosophes d'en chercher la cause mécanique; car ceux qui ne veulent point admettre dans la Philosophie des miracles perpétuels, doivent rendre raison des effets, par l'essence des choses et par le mouvement, et tout ce qui n'est point explicable par ces principes, n'est point du ressort de la Philosophie, qui ne doit s'occuper que des effets naturels, qu'on doit concevoir distinctement et expliquer intelligiblement."

62. Christia Mercer, Leibniz's Metaphysics, Cambridge, Cambridge University Press, 2001 . 